\title{
Separation of orbital contributions to the optical conductivity of $\mathrm{BaVS}_{3}$
}

\author{
I. Kézsmárki, ${ }^{1}$ G. Mihály, ${ }^{1}$ R. Gaál, ${ }^{2}$ N. Barisisić, ${ }^{2}$ A. Akrap,${ }^{2}$ H. Berger,${ }^{2}$ L. Forró, ${ }^{2}$ C.C. Homes,${ }^{3}$ and L. Mihály ${ }^{1,4}$ \\ ${ }^{1}$ Electron Transport Research Group of the Hungarian Academy of Science and Department of Physics, \\ Budapest University of Technology and Economics, 1111 Budapest, Hungary \\ ${ }^{2}$ Ecole Politechnique Federale, Lausanne, CH-1015 Switzerland \\ ${ }^{3}$ Condensed Matter Physics and Materials Sciences Department, \\ Brookhaven National Laboratory, Upton, NY 11973 USA \\ ${ }^{4}$ Department of Physics and Astronomy, Stony Brook University, Stony Brook, NY 11794-3800
}

(Dated: November 11, 2018)

\begin{abstract}
The correlation-driven metal-insulator transition (MIT) of $\mathrm{BaVS}_{3}$ was studied by polarized infrared spectroscopy. In the metallic state two types of electrons coexist at the Fermi energy: The quasi $1 D$ metallic transport of $A_{1 g}$ electrons is superimposed on the isotropic hopping conduction of localized $E_{g}$ electrons. The "bad-metal" character and the weak anisotropy are the consequences of the large effective mass $m_{e f f} \approx 7 m_{e}$ and scattering rate $\Gamma \geq 160 \mathrm{meV}$ of the quasi-particles in the $A_{1 g}$ band. There is a pseudo-gap above $T_{M I}=69 \mathrm{~K}$, and in the insulating phase the gap follows the BCS-like temperature dependence of the structural order parameter with $\Delta_{c h} \approx 42 \mathrm{meV}$ in the ground state. The MIT is described in terms of a weakly coupled two-band model.
\end{abstract}

PACS numbers:

In metals with low-dimensional electronic structure, Fermi surface instabilities can drive the system to various kinds of symmetry-breaking insulating ground state like a charge density wave (CDW) or a spin density wave (SDW). Although the primary force of these transitions is the Fermi surface instability, the ordered state often involves a lattice distortion. According to recent X-ray studies [1, 2], the MIT at $T_{M I}=69 \mathrm{~K}$ in the vanadium chain compound $\mathrm{BaVS}_{3}$ is also accompanied by the lowering of the crystal symmetry with four $\mathrm{V}$ ions along the chains in the doubled unit cell. However, $\mathrm{BaVS}_{3}$ is far from being a run-of-the-mill CDW or SDW system. The high-temperature phase is categorized as a "bad metal" where the mean free path is in the order of the $\mathrm{V}-\mathrm{V}$ distance and the susceptibility follows a Curie-Weiss law. Below the MIT, the susceptibility and the electronic entropy drop with no signs of long range magnetic order down to a subsequent transition at $T_{x} \approx 30 \mathrm{~K}[\underline{3}]$.

According to ARPES experiments [4] and band structure calculations $[\underline{5},[6,6],[]$ the wide-band $1 D$ electron system coexists with quasi-localized $3 D$ electrons around the Fermi energy. The spin degree of freedom of the localized electrons, the coupling between the localized and delocalized electrons, and the possibility of orbital ordering make the system more complex. Although the suppression of the MIT line and an adjacent quantumcritical region has been mapped by high-pressure experiments 9, 10, 11], there is still no generally accepted explanation for the MIT in this compound. The goal of the present optical study was to explore the anisotropic electronic excitations above and below the MIT.

Above $T_{M I}=69 \mathrm{~K}$ the unit cell contains two formula units and the formal valence of the vanadium ion is $3 d^{1}$. The two electrons per unit cell are distributed between two broad bands of $A_{1 g}$ symmetry, derived from vanadium $d_{z^{2}}$ orbitals with strong overlap along the chains $(c$ axis), and four narrow bands of $E_{a}$ symmetry, originating mostly from $e\left(t_{2 g}\right)$ orbitals [5, 6, 7, 8]. (For the structure of the material and labeling of the electronic orbitals see e.g. Ref. 12.) The electronic band structure in local density approximation shows that the two $A_{1 g}$ subbands (the "blue" bands in Fig. 1 of Ref. 8) are roughly symmetric to the Fermi energy with bonding- and antibonding-like character, hereafter referred to as $A_{1 g}$ and $A_{1 g}^{*}$, respectively. They have large dispersion along the $\Gamma Z$ line of the Brillouin zone and the low-lying $A_{1 g}$ branch crosses the Fermi level at the $Z$ point implying strongly anisotropic conduction with the $c$ axis as the best conducting direction. Recent ARPES experiments also indicate that two types of $\mathrm{V}$ bands $\left(A_{1 g}\right.$ and $\left.E_{g}\right)$ cross the Fermi energy [4]. Large on-site Coulomb repulsion is expected to introduce a correlation between electrons and lead to an approximately half-filled $A_{1 g}$ band [8].

Polarized reflectivity was studied on a high-quality oriented mosaic sample of $\mathrm{BaVS}_{3}$ in a photon energy range of $\hbar \omega=3 \mathrm{meV}-3.2 \mathrm{eV}$. The optical conductivity obtained by Kramers-Kronig transformation is denoted by $\sigma_{\|}$and $\sigma_{\perp}$ for polarizations parallel and perpendicular to the chain direction, respectively. The dc resistivity $\rho_{\|}$ was also measured on a high-quality $\mathrm{BaVS}_{3}$ crystal in an extended temperature range up to $T=650 \mathrm{~K}$. The $\rho_{\|}(T)$ curve is plotted in Fig. 1 together with the points derived from the optical conductivity according to $1 / \sigma_{\|}(\omega \rightarrow 0)$. The fairly good agreement between the two quantities confirms the validity of the data processing.

Fig. 2 shows the reflectivity and the wide-energy range conductivity spectra at representative temperatures for the two polarization directions. Above $\sim 1 \mathrm{eV}$ the optical conductivity is dominated by interband transitions leading to a broad, featureless contribution to both $\sigma_{\perp}(\omega)$ and $\sigma_{\|}(\omega)$. This continuum of absorption is approximately isotropic and assigned to various transitions be- 


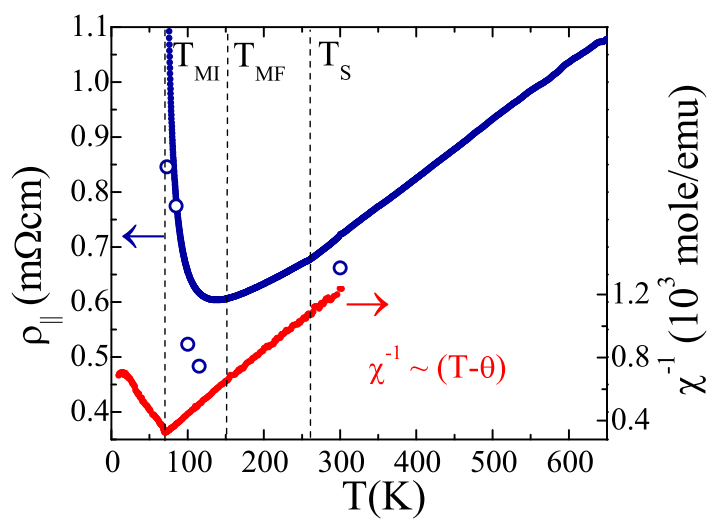

FIG. 1: (Color online) Temperature dependence of the resistivity $\rho_{\|}$(blue) and the inverse magnetic susceptibility $\chi^{-1}$ (red). Open circles corresponds to the zero-frequency extrapolation of the optical spectra according to $\rho_{\|} \equiv 1 / \sigma_{\|}(\omega \rightarrow 0)$.

tween strongly dispersive bands, including $\mathrm{S}(3 p) \rightarrow \mathrm{V}(3 d)$ electron transfer, with no particular preferred direction for the dipole matrix element. On this featureless background two peaks appear in $\sigma_{\|}(\omega)$ around $1.25 \mathrm{eV}$ and $2.5 \mathrm{eV}$. Based on the band structure calculations [5, 6, 7, 8] we assign the first peak to $E_{g} \rightarrow A_{1 g}^{*}$ and $S\left(\pi_{z}^{*}\right) \rightarrow E_{g}$ transitions. The former involves electron transfer between neighboring $\mathrm{V}$ ions and has large dipole matrix elements only for $E \| c$ since the $V-V$ distance is 3 times smaller along the chains than in the $a b$ plane. The joint density of states is also large, due to the flat dispersion of the bands between the $\Gamma-C-Y$ points. We note here that $S\left(\pi_{z}^{*}\right) \rightarrow A_{1 g}^{*}$ process may also give rise to similar anisotropic contribution probably corresponding to the smaller peak at $\sim 2.5 \mathrm{eV}[\underline{6}]$.

The analysis of the gap profile and the temperature dependence of the charge gap has been widely used to categorize the correlation-driven MITs 13. In the lowtemperature phase of $\mathrm{BaVS}_{3}$, there is a well-defined optical gap for both $E \| c$ and $E \perp c$. Gap values were determined by linearly extrapolating the steeply increasing edge of the spectra and taking the zero conductivity intercept as indicated in the enlarged plot of the lowenergy region in Fig. 3. Below $50 \mathrm{meV}$ strong phonon lines are visible: Several modes are exclusive to only one of the two polarizations, indicating the excellent orientation of the sample. Note that apart from the phonons, the optical conductivity vanishes below the gap energy.

The charge gap in the ground state is $\Delta_{c h}=42 \pm$ $5 \mathrm{meV}=470 \pm 60 \mathrm{~K}$ (in our convention $\Delta_{c h}=2 \Delta=E_{g}$ ). This yields an anomalously large gap ratio, $\Delta_{c h} / T_{M I} \approx$ 6. Nevertheless, the temperature dependence of the gap is close to a BCS-like behavior and matches well that of the structural order parameter (amplitude of the tetramerization along the $\mathrm{V}$ chains) as illustrated in Fig. 4(a). Gaps for the two polarizations are close, though the values determined from $\sigma_{\perp}(\omega)$ are some-

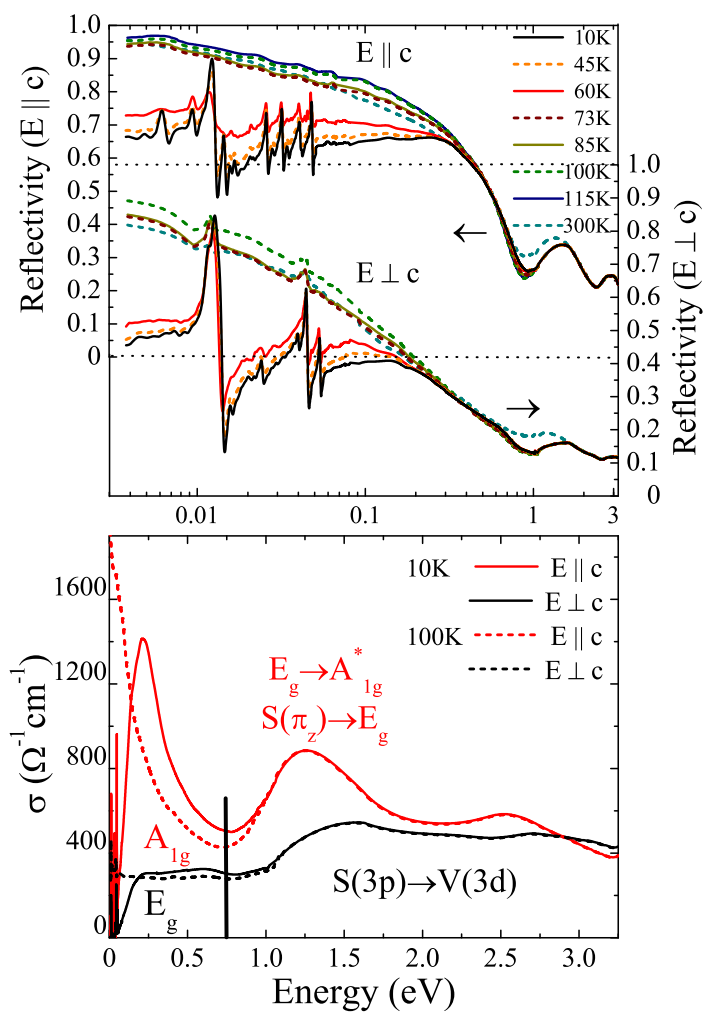

FIG. 2: (Color) Upper panel: Reflectivity spectra of $\mathrm{BaVS}_{3}$ at temperatures below $300 \mathrm{~K}$ for $E \| c$ and $E \perp c$. Lower panel: Optical conductivity at $T=10$ and $100 \mathrm{~K}$ with polarization parallel (red) and perpendicular (black) to the $c$ axis. Labels show the assignment of the different contributions.

what higher. Recently, Mitrovic et al. [4] estimated the ground-state gap by the leading-edge shift of the highresolution $(15 \mathrm{meV})$ photoemission spectra and found $\Delta_{c h}=60-70 \mathrm{meV}$. The estimates based on resistivity measurements 12, 14, 15 fall in a similar range, but they show large variation likely due to the different quality of the samples. Our optical study allows the first direct and precise observation of the charge gap in $\mathrm{BaVS}_{3}$.

Above the MIT a finite low-energy conductivity develops. For polarization along the chains $(E \| c)$ the phonon lines disappear, as expected due to the screening by conduction electrons. However, a pseudo-gap feature (a reduced conductivity at low frequencies) can be still discerned at $T=73 \mathrm{~K}$ and $85 \mathrm{~K}$ and the onset of coherent conduction is observed only at higher temperatures. The presence of the pseudo-gap is in agreement with several observations related to the $1 D$ fluctuations in this material, including the diffuse X-ray scattering [2] seen below $\sim 160 \mathrm{~K}$, the upturn both in the resistivity (see Fig. 1) and the Hall coefficient 10], and the appearance of an extra contribution in the thermoelectric power [16] below $\sim 120-140 \mathrm{~K}$. According to the well-established quasi $1 D$ scenario, the MIT is suppressed by fluctuations, and the "mean field" transition temperature is much higher than 


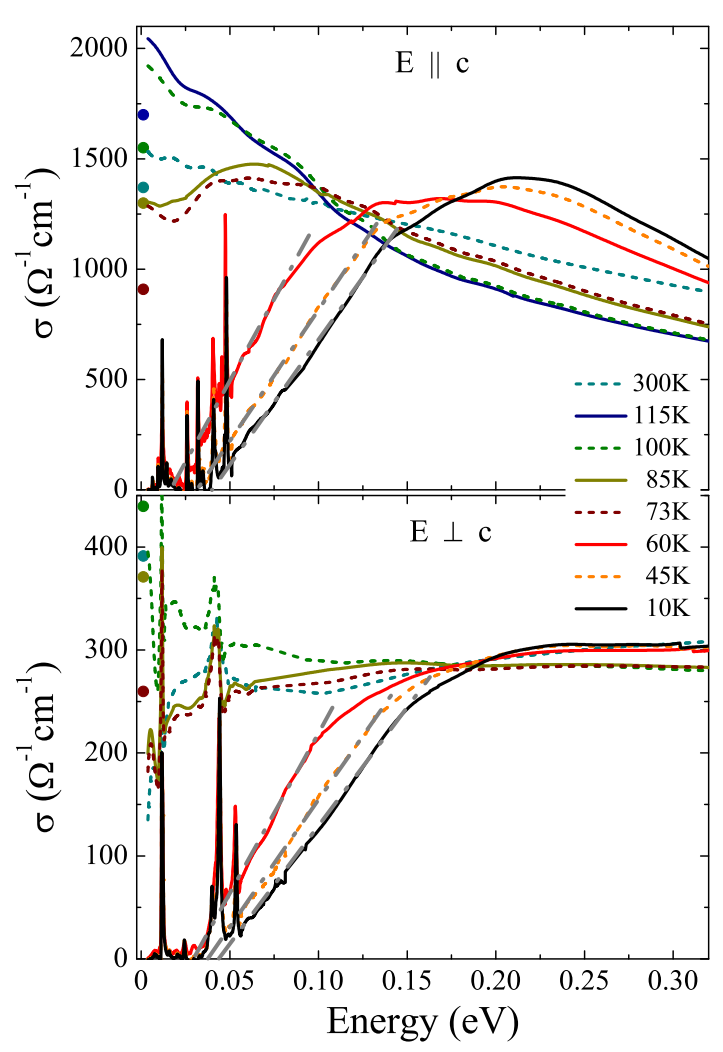

FIG. 3: (Color) Low-energy optical conductivity for $E \| c$ (top) and $E \perp c$ (bottom). Full circles represent the dc conductivity at the same temperatures obtained from resistivity measurements. For $T<T_{M I}$ the extrapolation to estimate the gap energy is shown by dash dotted grey lines. Note that the scales for $\sigma_{\|}$and $\sigma_{\perp}$ differ by a factor of $\sim 5$.

$T_{M I}=69 \mathrm{~K}$, probably in the $T_{M F}=120-160 \mathrm{~K}$ range. Indeed, with $T_{M F}$ the gap ratio is close to the BCS value of $\Delta_{c h} / T_{M F} \approx 3.5$. In quasi $1 D$ compounds exhibiting charge-ordering Peierls transition $\left(\mathrm{Ta}\left(\mathrm{Se}_{4}\right)_{2} \mathrm{I}, \mathrm{K}_{0.3} \mathrm{MnO}_{3}\right.$ [17] and $\mathrm{NbSe}_{3}[18]$ ), opening of the charge gap is also observed in the ordered phase. However, unlike in $\mathrm{BaVS}_{3}$, in these materials there is always a residual conductivity below the gap energy, attributed to $1 D$ fluctuations.

The metallic conduction with a Drude-like energy dependence is recovered along the chains around $100 \mathrm{~K}$, where $\rho_{\|}(T)$ has a minimum (see Fig. 1). At elevated temperatures the coherence is gradually lost and at room temperature the separation of a coherent contribution becomes ambiguous. In the metallic phase the lowfrequency optical conductivity perpendicular to the $c$ axis is $\sim 5$ times smaller than parallel to it, in agreement with the dc anisotropy $\sigma_{\|} / \sigma_{\perp} \approx 4\left[\underline{5}, 12\right.$. Furthermore, $\sigma_{\perp}(\omega)$ is practically independent of the frequency, apart from the phonon resonances that are still visible at room temperature. The low conductivity, the absence of coherence peak and the ineffective screening of phonon modes all imply an incoherent hopping conduction in the $a b$ plane.

We interpret the low-frequency optical data in terms of a "two-band" model. The $A_{1 g}$ band has strong dispersion along the $c$ direction, and carries the electrons responsible for the metallic character. The conductivity due to these carriers is expected to be strongly anisotropic - we will assume that their contribution is negligible in the directions perpendicular to $c$. On the other hand, the $E_{g}$ electrons are localized and they form flat bands. Their hopping conduction is essentially isotropic, since the molecular orbitals responsible for these bands are tilted relative to the principal crystallographic axes (their lobes point to the face and the edge center of the sulfur octahedra), and the orbital overlaps do not prefer any particular direction. Based on these simple assumptions, one can deduce the conductivity of the metallic band as $\sigma_{A_{1 g}}=\sigma_{\|}-\sigma_{E_{g}}$ where $\sigma_{E_{g}}=\sigma_{\perp}$. The resulting $\sigma_{A_{1 g}}(\omega)$ can be well fitted by a single Drude term, as presented in Fig. 4(b) for $T=115 \mathrm{~K}$. The effective number of electrons contributing to the Drude peak is $N^{e f f} \approx 0.072$ and their scattering rate is $\Gamma \approx 160 \mathrm{meV}$.

This interpretation is in agreement with other measurements, and sheds a new light to the long standing puzzle of $\mathrm{BaVS}_{3}$, how the low conduction anisotropy is realized in a metal with quasi $1 D$ crystal structure. The small $N^{e f f}$ for the conduction band leads to a large effective mass: Assuming $1 / 2$ electrons per vanadium in the $A_{1 g}$ subband [8] we obtain $m_{e f f} \approx 7 m_{e}$. The large overall bandwidth of $A_{1 g}$ electrons, estimated by band structure calculations $[5,6,67,[8]$ and determined by angle-resolved photoemission spectroscopy [4], would suggest a smaller mass. However, hybridization with $\mathrm{S}(3 p)$ subbands renormalizes the band mass that is further increased by correlation effects among which on-site Coulomb interaction is probably the most relevant. Although the $A_{1 g}$ electrons are metallic, their large mass combined with short relaxation time $(\tau \approx 0.026 \mathrm{ps})$ yields a low conductivity characterized by a mean free path close to the lattice constant 12 . The relatively weak anisotropy follows from the fact that the isotropic hopping conduction of the $E_{g}$ electrons is not negligible relative to the quasi $1 D$ metallic conduction of the $A_{1 g}$ band.

The separation of the different orbital contributions to the optical conductivity indicates weak coupling between the $A_{1 g}$ and $E_{g}$ electrons in the "bad-metal" phase of $\mathrm{BaVS}_{3}$. This picture is supported by the magnetic susceptibility which follows the Curie-Weiss law above $T_{M I}$, hence it is fully attributed to the localized $E_{g}$ electrons. Fig. 1 shows that there is no remarkable deviation from the linearity in $1 / \chi(T)$ down to the close vicinity of the MIT, indicating the absence of precursor fluctuations in the $E_{g}$ spin sector. In contrast, the in-chain transport coefficients governed by the $A_{1 g}$-type electrons fully reflect the $1 D$ structural fluctuations in the broad range $T_{M I}<T<140 \mathrm{~K}$. On this basis, we conclude that the primary force of the transition is the $1 D$ nature of the compound and thus the formation of CDW in the $A_{1 g}$ channel. The first manifestation of a finite coupling be- 

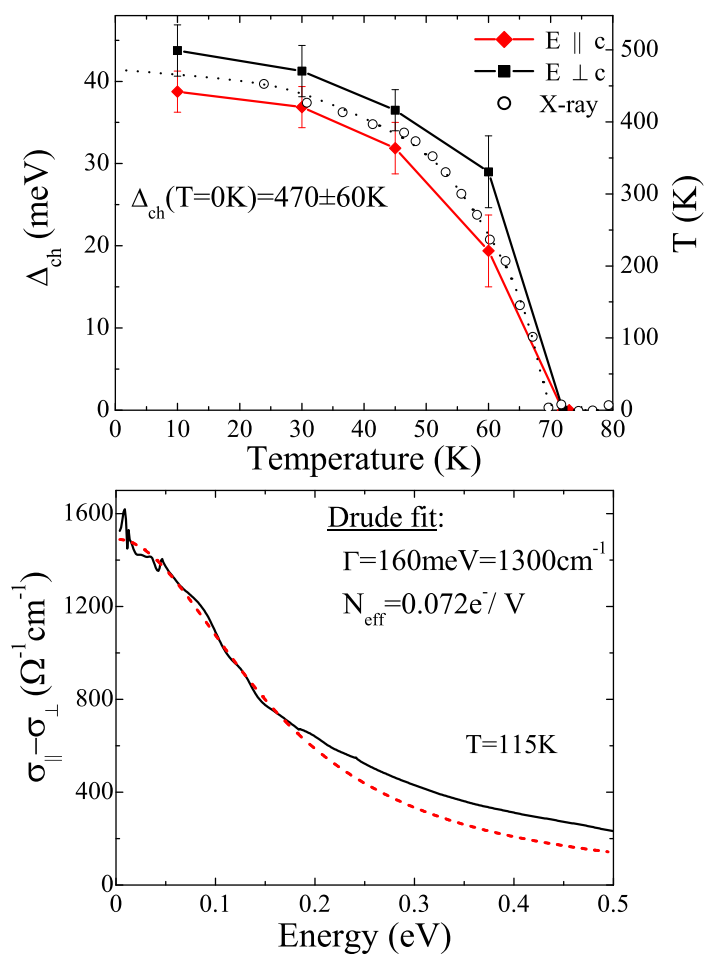

FIG. 4: (Color online) Top panel: Temperature dependence of the charge gap in the insulating phase of $\mathrm{BaVS}_{3}$ as determined from the optical conductivity (full symbols), and the structural order parameter (open symbols, on an arbitrary vertical scale) reproduced from Ref. 1, 2. The dashed line corresponds to the BCS gap profile. Bottom panel: Low-energy conductivity of the $A_{1 g}$-type $\mathrm{V}(3 d)$ electrons in the metallic phase of $\mathrm{BaVS}_{3}$ at $T=115 \mathrm{~K}$ evaluated according to $\sigma_{A_{1 g}}=\sigma_{\|}-\sigma_{\perp}$ (see text for details). The dashed line indicates the Drude fit.

tween the two kinds of electrons is the susceptibility cusp at $T_{M I}$ showing that $E_{g}$ electrons are also involved in the transition. The present results clearly evidence that the two sub-systems undergo a simultaneous transition, even with a similar gap value in the ground state. The MIT is accompanied by a large spectral weight transfer in $\sigma_{A_{1 g}}(\omega)$ from the Drude term to the correlation peak (the sharp structure centered at $\sim 0.25 \mathrm{eV}$ corresponding to excitations through the gap), while the small spectral weight shifted by the opening of the gap in $\sigma_{E_{g}}(\omega)$ is redistributed over a broader range of energy. The freezing out of $1 D$ lattice fluctuations, i.e. the lack of a subgap tail in $\sigma_{\|}(\omega)$ indicates the role of $E_{g}$ electrons in stabilizing the insulating state.

Recent X-ray experiments indicate that even in the tetramerized phase the valence of the vanadium ions is uniform, i.e. $\mathrm{V}^{4+}$ 19]. Apparently, the charge modulation in the $A_{1 g}$ channel is compensated by $E_{g}$ electrons. In a sense, this is natural in a system where the Coulomb correlation energies are large $(\sim 1-2 \mathrm{eV})$, and even the localized electrons has an orbital degree of freedom. Since the approximately two $E_{g}$ electrons in the tetramerized unit cell preferably occupy neighboring sites around the minima of the density wave, they will be susceptible of singlet formation. This "confinement of $E_{g}$ electrons by the CDW" explains the drop of the susceptibility below $T_{M I}$, and the development of a spin gap of $\Delta_{s} \approx 20 \mathrm{meV}$ $[3,11,20]$. The weak coupling between $E_{g}$ spins in the metallic state (reflected in the small Weiss temperature $\left.\Theta<10 \mathrm{~K} \ll T_{M I}\right)$ is in agreement with this picture: they have a tendency to form singlets, but the actual phase transition is not governed by this energy scale.

In conclusion, we have studied the charge dynamics in the vicinity of the correlation-driven MIT in the vanadium chain compound $\mathrm{BaVS}_{3}$ by polarized infrared spectroscopy. The unambiguous separation of the different orbital contributions to the low-energy optical conductivity in the metallic state allows the characterization of two types of electrons coexisting around the Fermi energy. The quasi $1 D$ metallic transport of $A_{1 g}$ electrons along the chains is superimposed on the isotropic hopping conduction of localized $E_{g}$ electrons. The "badmetal" character and the weak anisotropy are the consequence of the large effective mass $\left(m_{e f f} \approx 7 m_{e}\right)$ and the ill-defined nature of the quasi-particles in the $A_{1 g}$ conduction band. The latter is clearly manifested in the anomalously high scattering rate $\Gamma \geq 160 \mathrm{meV}$ almost comparable to the bandwidth. For $T_{M I}<T<100 \mathrm{~K}$ the onset of a pseudo-gap is observed while in the insulating phase the charge gap follows the temperature dependence of the structural order parameter as the $1 D$ lattice fluctuations are quenched. We believe that a weakly coupled model of itinerant $A_{1 g}$ and localized $E_{g}$ electrons can give a comprehensive description of $\mathrm{BaVS}_{3}$.

We are indebted to M. Miljak for the susceptibility measurements. Discussions with P. Fazekas, P. Thomas, I. Kupĉić and S. Bariŝić are gratefully acknowledged. L.M. received the Szent-Györgyi Fellowship from the Hungarian Ministry of Education and I.K. was a grantee of Bolyai János Fellowship. This work was supported by the Hungarian Research Founds OTKA TS049881, by the Swiss NSF, and its NCCR "MaNEP" and by the DOE under contract number DE-AC02-98CH10886.

[1] T. Inami et al., Phys. Rev. B 66, 73108 (2002).

[2] S. Fagot et al., Phys. Rev. Lett. 90, 196401 (2003).

[3] H. Nakamura et al., J. Phys. Soc. Jpn. 69, 2763 (2000).

[4] S. Mitrovic et al., cond-mat/0502144 v1 5 Feb 2005.

[5] L.F. Mattheiss, Solid State Commun. 93, 791 (1995).

[6] M.H. Whangbo et al., J. Solid State Chem. 165, 345 (2002).

[7] M.H. Whangbo et al., J. Solid State Chem. 175, 384 (2003).

[8] F. Lechermann, S. Biermann, and A. Georges, Phys. Rev. Lett. 94, 166402 (2005).

[9] L. Forró et al., Phys. Rev. Lett. 85, 1938 (2000).

[10] C.H. Booth et al., Phys. Rev. B 60, 14852 (1999). 
[11] I. Kézsmárki et al., Phys. Rev. B 63 (R)81106 (2001).

[12] G. Mihaly et al., Phys. Rev. B 61, (R)7831 (2000).

[13] M. Imada, A. Fujimori, Y. Tokura, Rev. Mod. Phys. 70, 1039 (1998).

[14] T. Graf et al., Phys. Rev. B 51, 2037 (1995).

[15] M. Nakamura et al., Phys. Rev. B 49, 16191 (1994).

[16] L. Forró et al., to be published.

[17] L. Degiorgi et al., Phys. Rev. B 52, 5603 (1995).
[18] A. Perucchi, L. Degiorgi, R.E. Thorne, Phys. Rev. B 69, 195114 (2004).

[19] S. Fagot, P. Foury-Leylekian, S. Ravy, J.P. Pouget, E. Lorenzo, Y. Joly, M. Greenblatt, M.V. Lobanov and G. Popov, Phys. Rev. B 73, 033102 (2006).

[20] H. Nakamura, H. Imai, M. Shiga, Phys. Rev. Lett. 79, 3779 (1997). 